\title{
Changes in the mechanical response of dental stone to submersion in saline
}

Joanne Cai ${ }^{1}$, Dan Romanyk, Kathryn Houg

${ }^{1}$ Department of Mechanical Engineering, University of Alberta

\begin{abstract}
Previously, dental stone has been utilized in mechanical testing of the periodontal ligament, which connects the tooth to the alveolar bone. This was achieved by casting swine mandibles in dental stone for displacement controlled testing. The stone was used as a riding base to hold the mandible in place. However, this was done in dry, ex vivo conditions, failing to simulate the natural presence of vascular fluid in the periodontal ligament. While this can be simulated with submersion in saline, the mechanical response of dental stone to saline is currently unknown, and thus cannot yet be used to secure the mandible in saline. To identify differences in the strength of dental stone after submersion in saline, Coecal Type III Dental Stone samples were cast in a 3D printed mold, then submerged in $0.9 \% \mathrm{NaCl}$ solution for varying time periods: 0 hours, 0.5 hours, 1 hour, 4 hours, and 24 hours. Samples were then removed from the solutions, patted dry, and placed in an Instron ElectroPuls E3000 for compression tests. The Instron preloaded samples to $3 \mathrm{~N}$, displaced them to $0.1 \mathrm{~mm}$ at $0.025 \mathrm{~mm} / \mathrm{s}$, held them for $10 \mathrm{~s}$, then offloaded at $0.025 \mathrm{~mm} / \mathrm{s}$. This cycle was repeated five times per test, with each sample undergoing two tests. Results showed that the last three cycles of each test were most consistent, and were the only ones considered in further analysis of results. The average peak force and average force during the $10 \mathrm{~s}$ hold of each test were compared across submersion times, and, considering standard deviation, showed no consistent differences. A linear regression was completed to determine statistical differences between the force values. The $p$ values of the average peak force and average force were 0.624 and 0.892 respectively, approaching the required value of 1 , and therefore failed to reject the null hypothesis, representing no significant difference in the average force across submersion times. As a result, it can be assumed that submersion in saline has no effect on the strength of dental stone. These results can be applied to further research on the periodontal ligament, involving submersion of the swine mandible in saline solutions.
\end{abstract}

Key words:

dental stone, saline, mechanical response, dental stone strength, submersion

Cite as: Cai J., Romanyk D., and Houg K. 2019. Changes in the mechanical response of dental stone to submersion in saline. Alberta Academic Review, Vol 2 (2) 19-20, WISEST Special Issue (non peer-reviewed), DOI 10.29173/aar55. 
Cai et al., 2019

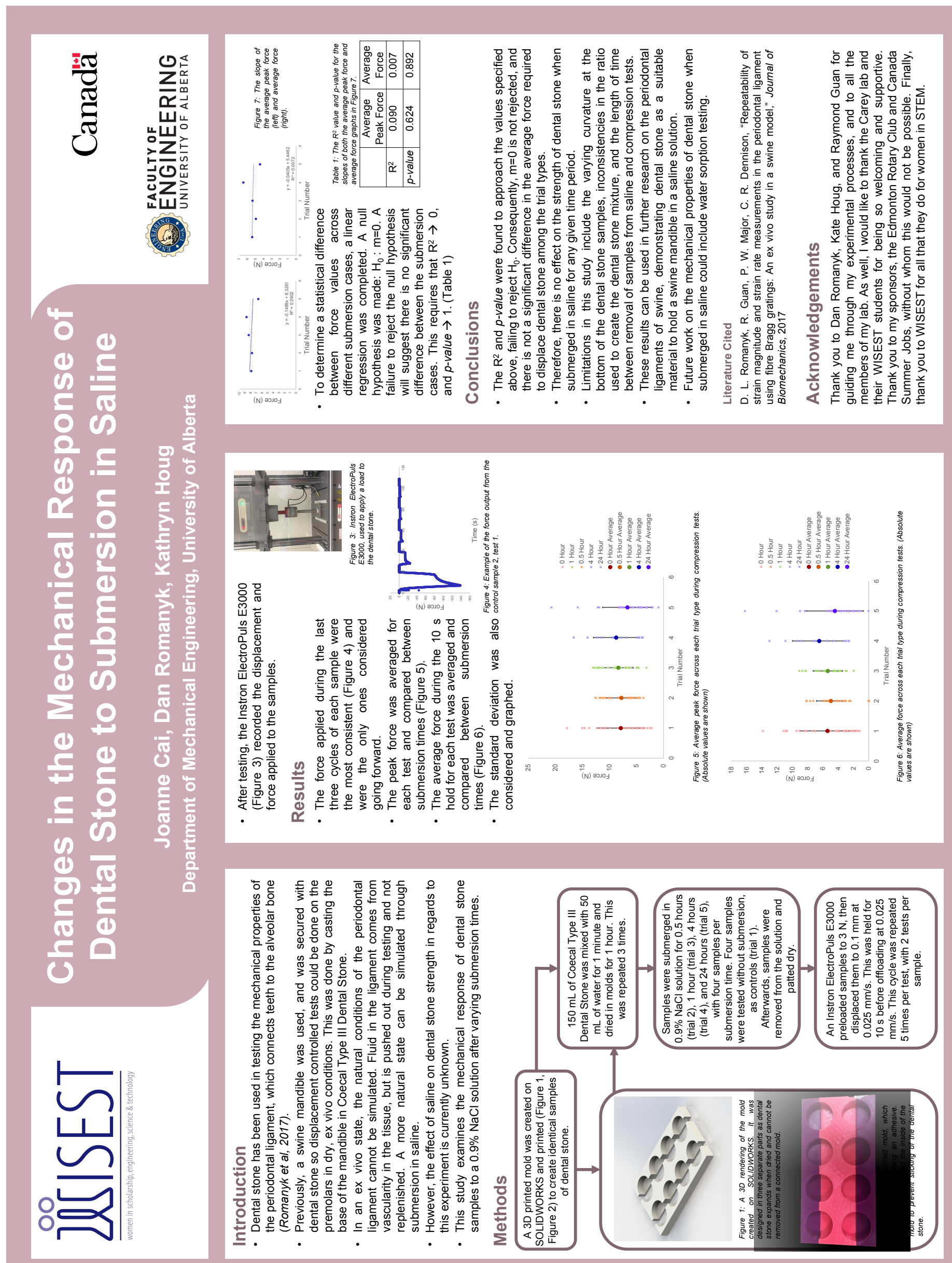

